\title{
PROBLEMAS NA RECUPERAÇÃO DE PACIENTES SUBMETIDOS À CIRURGIA DE REVASCULARIZAÇÃO DO MIOCÁRDIO: O ACOMPANHAMENTO PELO ENFERMEIRO DURANTE O PRIMEIRO MÊS APÓS A ALTA HOSPITALAR
}

Rosana Aparecida Spadoti Dantas ${ }^{1}$

Olga Maimoni Aguillar ${ }^{2}$

Dantas RAS, Aguillar OM. Problemas na recuperação de pacientes submetidos à cirurgia de revascularização do miocárdio: 0 acompanhamento pelo enfermeiro durante o primeiro mês após a alta hospitalar. Rev Latino-am Enfermagem 2001 novembro-dezembro; 9(6):31-6.

Pacientes após revascularização do miocárdio podem apresentar problemas e os enfermeiros têm importante papel na deteç̧ão dos mesmos. Com o objetivo de determinar problemas apresentados por 25 sujeitos no primeiro mês após a alta hospitalar optamos por seguí-los por contatos telefônicos e no retorno ambulatorial. $A$ análise dos relatos resultou em oito categorias. Os problemas mais freqüentes estiveram relacionados à ferida cirúrgica (76\%), à terapêutica medicamentosa (28\%), à outras patologias ((24\%), à alterações do humor (20\%) e ao sono (16\%). A estratégia utilizada foi eficaz e permitiu acompanhar a recuperação dos doentes no primeiro mês após a alta.

DESCRITORES: revascularização miocárdica, recuperação

\section{PROBLEMS DURING THE RECOVERY OF PATIENTS FOLLOWING CORONARY ARTERY BYPASS SURGEY: THE FOLLOW-UP BY NURSES IN THE FIRST MONTH AFTER HOSPITAL DISCHARGE}

Patients may present problems after coronary artery bypass surgery and nurses play an important role in the detection of such problems. With the purpose to determine the problems of 25 patients during the first month after hospital discharge, they were followed up via telephone as well as when they returned to hospital. Content analyses of detailed nurses' recording revealed eight categories of problems. The most frequently related problems were related to the surgical incision site (76\%), pharmacological therapy (28\%), other pathologies (24\%), mood alterations (20\%) and sleep pattern disturbances (16\%). The strategy chosen showed to be efficient to analyze the recovery of these patients during the first month after hospital discharge.

KEY WORDS: coronary artery bypass surgery, recovery

\section{PROBLEMAS EN LA RECUPERACIÓN DE PACIENTES SOMETIDOS A CIRUGÍA DE REVASCULARIZACIÓN DEL MIOCARDIO: SEGUIMIENTO DE ENFERMERÍA EN EL PRIMER MES DESPUES DE LA ALTA HOSPITALARIA}

Tras la cirugía de revascularización del miocardio los pacientes pueden presentar problemas y los enfermeros tienen un importante papel en la detección de los mismos. Con el objetivo de determinar problemas presentados por 25 pacientes en el primer mes después del alta hospitalaria, optamos por hacerles un seguimiento mediante contactos telefónicos y durante su retorno al hospital. El análisis de sus relatos indicó ocho categorías. Los problemas más frecuentes estuvieron relacionados con la herida quirúrgica, el tratamiento farmacológico (28\%), otras enfermedades (24\%), alteraciones del humor (20\%) y cambios en el patrón de sueno (16\%). La estrategia escogida fue eficaz y permitió acompañar la recuperación de estos pacientes durante el primer mes después del alta hospitalaria.

\section{DESCRIPTORES: revascularización miocardica, recuperación}

${ }^{1}$ Enfermeiro, Professor Doutor da Escola de Enfermagem de Ribeirão Preto da Universidade de São Paulo, Centro Colaborador da OMS para o desenvolvimento da pesquisa em enfermagem, e-mail: rsdantas@eerp.usp.br; ${ }^{2}$ Enfermeiro, Professor Doutor da Escola de Enfermagem de Ribeirão Preto da Universidade de São Paulo, Centro Colaborador da OMS para o desenvolvimento da pesquisa em enfermagem 
INTRODUÇÃOO

tratamento dos indivíduos, portadores de doença isquêmica cardíaca, vem sofrendo avanços terapêuticos, clínicos e cirúrgicos nos últimos 30 anos $^{(1)}$. A cirurgia de revascularização do miocárdio é uma das opções no tratamento cirúrgico destes indivíduos e tem como objetivos: prolongar a vida, promover alívio da dor de angina e melhorar a qualidade de vida dos pacientes ${ }^{(1-2)}$.

Por ser procedimento cirúrgico de grande porte os pacientes estão sujeitos a vários tipos de complicações nos períodos trans e pós-operatório. Complicações cardiovasculares, pulmonares, renais, gastrointestinais e neuropsiquiátricas são possíveis de ocorrer ${ }^{(3-5)}$. Estas complicações ocorrem em 15\% dos indivíduos que são submetidos à cirurgia de revascularização do miocárdio (RVM). Pacientes que tiveram uma ou mais complicações têm mortalidade pós-operatória entre 8 a 10\% maior que aqueles que não as desenvolveram ${ }^{(6)}$. Os principais fatores predisponentes para as complicações perioperatórias são: idade avançada, doença vascular periférica, creatinina sérica elevada, sexo feminino, diabetes e diálise pós-operatória ${ }^{(6-7)}$. Quando tais complicações ocorrem, os custos para os pacientes são incalculáveis tanto do ponto de vista financeiro como físico e emocional.

Visando a contenção de gastos hospitalares, a alta dos pacientes após cirurgia cardíaca tem sido cada vez mais precoce. Tal conduta pode ter efeito contrário sobre a proposta de contenção de custos e a re-hospitalização pode ser um dos resultados. Estudos têm demostrado que as causas mais comuns para a reinternação dos doentes são: arritmias, problemas respiratórios, derrame pleural, insuficiência cardíaca congestiva e problemas trombo-embólicos. Presença de dor torácica, com ou sem comprometimento respiratório, problemas incisionais, alterações gastrointestinais e efeitos adversos dos medicamentos, como naúseas, vômitos e dores gástricas também foram constatados ${ }^{(8-9)}$. Outros estudos identificaram também problemas relacionados à ferida cirúrgica, terapêutica medicamentosa, mudanças nos padrões de sono, bem como alterações no apetite, mudanças emocionais e disfunções neuromusculares ${ }^{(3-5,10-12)}$

Com a diminuição do tempo de hospitalização, o planejamento de um programa de acompanhamento dos pacientes tornou-se essencial. $\mathrm{O}$ uso do telefone para tal seguimento tem sido estudado por vários autores ${ }^{(5,13-15)}$. O uso deste recurso é uma maneira de propiciar aos mesmos rápido acesso ao serviço de saúde e de fornecer aos doentes e familiares aquilo que eles estão necessitando em termos de orientação e apoio. Com esta possibilidade de acompanhamento eles podem procurar por orientação no momento em que sentirem necessidade ${ }^{(16-17)}$.

O retorno para casa após a alta hospitalar é um momento de ansiedade para o paciente e familiares, uma vez que se sentem desprotegidos da vigilância constante da equipe de saúde fora do hospital. Assim, a alta hospitalar pode ser vista como uma ameaça para suas vidas ${ }^{(18)}$. O primeiro mês de recuperação é particularmente estressante para o doente e família. Eles podem reportar ansiedade, nervosismo, depressão, fadiga, distúrbios no sono, mudanças no apetite, desconforto nas incisões, dificuldades respiratórias e presença de dores na região torácica. Dor e desconfortos nas incisões cirúrgicas, bem como dores musculares nos ombros, costas e pescoço, levam os mesmos a evitarem movimentos respiratórios profundos e se exercitarem físicamente ${ }^{(19-21)}$.

A recuperação de 125 indivíduos revascularizados foi acompanhada por três meses, através de chamadas telefônicas feitas por iniciativa dos doentes. De um total de 217 chamadas, $40 \%$ delas ocorreram na primeira semana após a alta e $15 \%$, na segunda semana. Os problemas mais relatados foram distúrbios cardiopulmonares (como dor torácica), dor incisional, edema em membros inferiores, distúrbios gastrointestinais (p. ex. diarréia) e diminuição da atividade física. Das 217 chamadas, 159 foram de pacientes após cirurgia cardíaca e 58 com tratamento clínico ${ }^{(16)}$.

Muitas dúvidas poderão surgir nos primeiros dias após a alta tais como questões relacionadas ao tratamento, aparecimento e/ou manutenção de sinais e sintomas e surgimento de novos problemas. Acreditamos que, nos primeiros meses após a cirurgia, 0 enfermeiro tenha um importante papel na assistência junto ao paciente revascularizado e seus familiares. Esta assistência pode estar centrada em cuidados como curativos, administração de medicamentos, fisioterapia e educação visando a sua recuperação e detecção de problemas.

A pesquisa que apresentamos neste texto faz parte de uma investigação mais ampla que abordou o acompanhamento de pacientes submetidos 'a cirurgia de RVM, durante os seis primeiros meses após a alta. $O$ recurso escolhido para o seguimento foi o uso de chamadas telefônicas e o acompanhamento ambulatorial. Durante esta investigação, os sujeitos participaram de um protocolo de assistência de enfermagem voltado para a reabilitação de doentes cardíacos. 0 protocolo de enfermagem desenvolvido e as ações de enfermagem realizadas diante dos problemas levantados durante 0 acompanhamento dos doentes podem ser encontrados no estudo que originou este artigo ${ }^{(22)}$.

Esta pesquisa teve como objetivo identificar os problemas apresentados pelos pacientes, submetidos à cirurgia de RVM, no primeiro mês após a alta. 


\section{MÉTODOS}

O estudo foi realizado em um hospital escola do interior de São Paulo, no período de janeiro a junho de 1998, após aprovação pelo Comitê de Ética da instituição hospitalar. 0 grupo estudado constou de 25 pacientes submetidos à cirurgia de revascularização do miocárdio, que obedeceram aos seguintes critérios de inclusão: não terem sido submetidos a outro procedimento no ato da cirurgia (como correção de valvulopatias); estarem em condições físicas e psicológicas para participar do programa educativo desenvolvido pela pesquisadora; terem telefone em suas residências ou em outro local de fácil acesso; concordarem em participar do estudo.

Durante o período de internação pós-operatório estabelecemos contato com os doentes e seus familiares e apresentamos os objetivos do estudo. Com a concordância dos mesmos, entregávamos o termo de consentimento esclarecido para a assinatura dos envolvidos ${ }^{(23)}$.

Após a aquiescência dos pacientes, desenvolvemos um programa educativo abordando os seguintes aspectos: a doença isquêmica cardíaca (causas, sinais e sintomas, prognóstico), o tratamento cirúrgico (número e tipo de enxertos colocados; processo de cicatrização normal e com sinais de infecção; cuidados com 0 curativo) e o processo de reabilitação após a alta hospitalar (tratamento medicamentoso e controle dos fatores de risco: alimentação, exercícios físicos, tabagismo).

Para o acompanhamento dos sujeitos após a alta hospitalar, utilizamos contatos por telefone e a participação durante as consultas ambulatoriais. Os resultados aqui apresentados irão abranger apenas o primeiro retorno ambulatorial, o qual ocorreu, em média, na quarta semana após a alta hospitalar. Os contatos telefônicos foram feitos em torno do décimo e vigésimo dias, porém dependendo das situações apresentadas, outros telefonemas foram feitos antes do retorno, tanto por iniciativa da enfermeira-pesquisadora quanto dos doentes e familiares. Tal acompanhamento visou detectar os problemas apresentados por estes pacientes quando retornaram ao lar e, ao mesmo tempo, assistir estes indivíduos e familiares enquanto um profissional da equipe de saúde, ou seja, um enfermeiro.

Para o levantamento e evolução dos aspectos clínicos dos participantes, elaboramos um roteiro com dados de identificação do doente, história clínica, cirurgia realizada e fatores de risco coronarianos presentes no estilo de vida. Dados antropométricos (peso, altura e índice de massa corporal), hemodinâmicos (pressão arterial e freqüência cardíaca) e bioquímicos (lipidograma e glicemia) também foram registrados. Tais informações foram coletadas no início do acompanhamento e a cada retorno ambulatorial. Além dos dados clínicos, foram levantadas informações do processo de reabilitação cardíaca e referentes ao retorno ao trabalho e à vida sexual, participação da família em sua recuperação e mudanças em seu estilo de vida. A cada contato, os dados obtidos eram atualizados no roteiro do paciente e novas notas de campo eram anexadas ao seu processo de acompanhamento.

Para esta coleta de dados, utilizamos de entrevistas abertas, nas quais eram feitas as seguintes questões norteadoras: Como você tem passado após a cirurgia? Como está sendo a sua recuperação? Não foi estipulado um roteiro pré-determinado para o encontro ambulatorial e para os contatos telefônicos uma vez que os aspectos discutidos nestas ocasiões eram aqueles trazidos pelos doentes e seus familiares e que diziam respeito ao processo de reabilitação. No entanto, durante os diálogos buscávamos investigar a presença daqueles problemas que são comuns nos primerios meses após a cirurgia ${ }^{(3-5,7,10-12,24-25)}$

Para o registro dos dados foi utilizada a técnica de "notas de campo"(26). Os relatos dos encontros e contatos telefônicos foram posteriormente analisados e os conteúdos referentes aos problemas apresentados foram agrupados em categorias. A análise do conteúdo das "notas de campo" seguiram as seguintes etapas: (1) documentação do processo de interação entre enfermeira e paciente e/ou familares e (2) descrição da freqüência e intensidade dos problemas apresentados, (3) descrição da conduta adotada pelo doente e pela enfermeira diante da detecção dos mesmos. Os problemas apresentados por indivíduos revascularizados e levantados nos estudos consultados ${ }^{(16,20-21)}$ serviram como pré-categorização dos possíveis resultados a serem encontrados.

\section{RESULTADOS}

Os 25 participantes eram predominantemente do sexo masculino (96\%), com idade entre 50 e 70 anos (68\%), casados (88\%) e procedentes da cidade e região (72\%). Quanto aos aspectos sócioeconômicos, $68 \%$ deles eram aposentados, mais de $50 \%$ não haviam completado o primeiro grau de escolaridade e tinham renda mensal familiar até 6 salários-mínimos.

Com relação ao processo cirúrgico, 16 (64\%) receberam enxerto misto (arterial e venoso), 7 (28\%) só enxerto arterial, 1 (4\%) venoso e 1 (4\%) enxerto heterólogo. Quanto ao número de enxertos realizados, 12 (48\%) receberam 3 pontes, 7 (28\%), 2 pontes, 4 (16\%), 1 ponte e os outros $2(8 \%), 4$ pontes. Apenas dois deles ( $8 \%$ ) foram operados em caráter de urgência. 0 tempo médio de internação, após a revascularização, foi de 9 dias, variando de 4 a 18 dias.

A Tabela 1 mostra os problemas apresentados pelos pacientes nos primeiros trinta dias após a alta hospitalar. 
Tabela 1 - Distribuição dos 25 pacientes revascularizados, segundo os problemas relatados nos primeiros 30 dias após a alta hospitalar*

\begin{tabular}{lcc}
\hline \multicolumn{1}{c}{ Problemas relacionados à } & $n$ & $\%$ \\
\hline Ferida cirúrgica & 19 & 76 \\
Terapêutica medic amentosa & 7 & 28 \\
Presença de outras patologias & 6 & 24 \\
Afteraçẫo do humor & 5 & 20 \\
Afteraçẫo do padrẫo de sono & 4 & 16 \\
Queixas cardiovasculares & 4 & 16 \\
Alteraçã̃o: sensibilidade, motricidade, & 3 & 12 \\
memória, acuidade visual e força muscular & & \\
After açẫo do apette & 2 & 8 \\
\hline
\end{tabular}

* houve pacientes que apresentaram mais de um problema

0 problema mais relatado esteve relacionado à ferida cirúrgica e referiu-se a presença de dor, edema, presença de secreção e sensação de calor nos locais das incisões cirúrgicas. Como o enxerto venoso utilizado é a veia safena, $17(68 \%)$ tiveram mais de uma incisão cirúrgica. Dezenove doentes $(76 \%)$ relataram um ou mais de um dos sinais e sintomas acima descritos. Seis $(24 \%)$ referiram que tais sintomas em membros inferiores tinham dificultado a realização das caminhadas diárias que haviam sido propostas pela equipe de saúde. Um paciente, diabético e obeso, apresentou infeção da incisão cirúrgica torácica e do mediastino tendo que ser re-hospitalizado.

$\mathrm{Na}$ alta hospitalar, os pacientes receberam a prescrição média de 3 medicamentos, sendo mais prescritos os anti-agregantes plaquetários (geralmente o ácido acetil-salicílico), os vasodilatadores coronarianos (principalmente os nitratos) e anti-hipertensivos. $\mathrm{O}$ uso de vários e diferentes medicamentos pode causar problemas para os doentes. Em nosso estudo, a terapêutica medicamentosa foi 0 segundo problema mais relatado. Eles queixaram-se do aparecimento de efeitos secundários como cefaléia (decorrente do uso de nitratos), desconfortos gástricos (relacionados ao uso de ácido acetil-salicílico), e de episódios de hipertensão ou hipotensão arterial e hipoglicemia (relacionadas à dosagem de anti-hipertensivos e hipoglicemiantes).

A presença de outras patologias, comprometendo o estado clínico dos participantes, foi o terceiro problema mais detectado. Doenças como litíase renal, problemas articulares e lesão ulcerosa em membros inferiores; infecções virais e colecistite foram relatados por 6 (24\%) participantes. Dois foram re-hospitalizados em decorrência de litíase renal e um terceiro foi submetido à cirurgia para retirada de cálculo na vesícula biliar.

Mudanças nos comportamentos e hábitos diários foram atribuídas à experiência cirúrgica pelos participantes. Entre eles, 5 $(20 \%)$ relataram mudanças no humor, $4(16 \%)$ no padrão de sono e $2(8 \%)$ no apetite. Aqueles que referiram alterações no humor, salientaram aumento da irritabilidade, sensação de desânimo e depressão após o retorno ao lar. Dor no local cirúrgico, principalmente, na incisão torácica, foi a causa atribuída à alteração no padrão de sono.
Alterações de sensibilidade ao redor da incisão cirúrgica, principalmente na região torácica, alterações no padrão de motricidade, com perda da força muscular, e, comprometimento de funções intelectuais, como a memória, foram relatadas por $3(12 \%)$ doentes. A presença de queixas cardiovasculares como cansaço, taquicardia aos esforços, precordialgia, dispnéia e ortopnéia foram relatadas por $4(16 \%)$ deles.

\section{DISCUSSÃO}

Em nosso estudo observa-se a presença de problemas referentes à ferida cirúrgica, à terapêutica medicamentosa, alterações no sono, apetite e humor, bem como queixas cardiovasculares. Os mesmos resultados foram constatados em estudos anteriores ${ }^{(11,20,27-}$ 29)

Problemas relacionados à ferida cirúrgica como pequenas deiscências, edema e infecções nas incisões de membros inferiores não são incomuns, porém costumam ser de pouca gravidade ${ }^{(24)}$. As complicações na incisão torácica são importantes causas de morbidade em pacientes submetidos à cirurgia cardíaca, via esternotomia mediana ${ }^{(7)}$. A dor referida pelos pacientes, submetidos à cirurgia cardíaca, é causada pela secção dos nervos intercostais, ao longo do trajeto da incisão e pela irritação da pleura devido a presença de drenos torácicos, diminuindo gradativamente nos 2 meses de recuperação ${ }^{(20,30)}$. Os pacientes do presente estudo atribuíam à dor na região torácica suas dificuldades para desenvolverem movimentos respiratórios amplos, tossir ou mesmo encontrar posições confortáveis para induzir e manter o sono. Assim, as preocupações e os problemas relacionados à ferida cirúrgica são aspectos importantes a serem abordados pelos enfermeiros junto aos pacientes além de ser um dos temas prioritários para a educação dos pacientes ${ }^{(31)}$

Outros problemas relacionados à terapêutica medicamentosa e às queixas mais freqüentes foram relacionados à presença de efeitos colateriais e desajustes de dosagens. A necessidade de informações com relação aos diferentes tipos de medicação utilizada, após a revascularização do miocárdio, foi constatada em outros estudos ${ }^{(11,27-28,32)}$.

Problemas relacionados à presença de outras patologias, compromentendo o estado geral dos participantes e dificultando a recuperação cirúrgica, não foram levantados em outros estudos ${ }^{(16,20-}$ 21)

Alterações no padrão de motricidade, com perda de força muscular e comprometimento das funções intelectuais podem ser ocasionadas por lesões neuro-musculares decorrentes de inúmeros fatores como anestesia, circulação extra-corpórea, medicamentos e 
alterações do padrão de sono. No entanto, tais disfunções tendem a ser temporárias e desaparecem entre 6 semanas a 6 meses, após a cirurgia ${ }^{(12,25)}$.

A incidência de sensação de cansaço, distúrbios do padrão de sono, mudanças no apetite também foram reportadas por pacientes revascularizados nas 3 primeiras semanas após alta ${ }^{(20)}$. Tais problemas foram observados em nosso estudo, durante o primeiro mês de recuperação. Com relação à alteração no padrão de humor, relatada no grupo estudado como tristeza, depressão e desânimo, alguns autores ${ }^{(33)}$ referem que no período pós-operatório do paciente revascularizado são comuns crises de choro e momentos de tristeza. É tempo de reflexão para o doente que costuma analisar sua vida anterior. A sensação de culpa e de ter provocado a sua doença cardíaca com comportamentos e hábitos pouco saudáveis, pode atormentar o paciente neste período ${ }^{(10)}$.

Alguns pacientes continuam a ter problemas, mesmo após terem se submetido a procedimento terapêutico, com sucesso ${ }^{(34)}$. Os programas de reabilitação cardíaca são importantes porque novos problemas serão manifestados, diferentes daqueles apresentados antes da doença ou do tratamento. A educação do paciente é um importante aspecto na assistência de enfermagem. 0 preparo do revascularizado para o auto-cuidado requer informações específicas sobre os problemas observados neste estudo e por outros autores ${ }^{(3,11,27-28,31,35)}$

Entre os enfermeiros que atuam em serviços de cirurgia cardíaca uma dúvida frequente é: Quais são os problemas enfrentados pelos pacientes quando deixam este hospital e retornam aos seus lares? A resposta para esta questão poderá ajudar a esclarecer um outra questão ainda mais complexa: Quais são as informações

\section{REFERÊNCIAS BIBLIOGRÁFICAS}

1. McGovern PG, Pankow JS, Sahar E, Doliszny KM, Folsom AR, Blackburn $\mathrm{H}$, et al. Recent trends in acute coronary heart disease: mortality, morbidity, medical care and risk factors. N Engl J Med 1996; 334(14):884-90.

2. Clancy CA, Wey JM, Guinn GA. The effect of patient's perception on return to work after coronary artery bypass surgery. Heart \& Lung 1984; 13(2):173-6.

3. Gregersen RA, McGregor MS. Cardiac surgery. In: Underhill SL, Woods, SL, Froelicher, ES, Halpeny, CJ, editors. Cardiac nursing. 2. ed. Philadelphia: J.B. Lippincont; 1989. p.537-60.

4. Isogro F, Schmidt C, Pohl P. A predictive parameter in patients with brain related complications after cardiac surgery. Eur J Cardiothorax Surg 1997; 11(4):640-4.

5. Verdi JMC, Saadi EK, Barbosa GV. Pré e pós operatório de cirurgia cardíaca. In: Barreto SM, organizador. Rotinas em terapia intensiva. Porto Alegre (RS): Artes Médicas; 1993. p. 278-85. realmente necessárias e que devem ser fornecidas ao doente e aos familiares antes da alta para que eles possam enfrentar, efetivamente, esta fase inicial da reabilitação cardíaca?

O enfermeiro, por ser um profissional com maior grau de proximidade com o paciente e familiares, está mais capacitado para avaliar o processo educativo, levantando as necessidades educacionais destes indivíduos, suas crenças e valores, 0 autoconhecimento de suas condições de saúde e serviços de apoio existentes para o processo de reabilitação dos pacientes ${ }^{(36)}$.

\section{CONSIDERAÇÕES FINAIS}

Constatamos, no decorrer deste estudo, a importância do seguimento dos indivíduos revascularizados nos primeiros 30 dias após a alta hospitalar. Tal importância esteve relacionada não somente ao paciente e aos familiares, mas também ao papel do enfermeiro e da instituição de saúde onde se realizou o tratamento cirúrgico.

Quanto ao paciente e familiares, consideramos que a possibilidade de poderem entrar em contato com a enfermeira, através de chamadas telefônicas, bem como por receberem telefonemas desta profissional, indagando sobre o seu estado de saúde, transmitiram confiança e tranqüilidade para eles enfrentarem o primeiro mês após a alta. Acreditamos que a estratégia de acompanhamento de pacientes, após a alta hospitalar, através de contatos telefônicos, seja um mecanismo valioso, e pouco oneroso, para se avaliar o processo de reabiltação dos doentes sob nossos cuidados.

6. Hammermeister KE, Burchfiel C, Johnson R, Grover FL. Identification of patients at greatest risk for developing major complications at cardiac surgery. Circulation 1990; 82(5 Suppl 4):3809.

7. Stáhle E, Tammelin A, Bergstrom R, Hambreus A, Nystrom SO, Hansson HE. Sternal wound complications - incidence, microbiology and risk factors. Eur J Cardiothorax Surg 1997; 11(6):1146-53.

8. Herlitz J, Albertsson P, Brandrup-Wognsen G, Emamuelsson H, Hartford M, Hjalmarson A, et al. Predictors of hospital readmission two years after coronary artery bypass grafting. Heart 1997 May; 77(5):437-42.

9. Sabourin CB, Funk M. Readmission of patients after coronary artery bypass graft surgery. Heart \& Lung 1999; 28(4):243-50.

10. Burke LE, Scalzi CC. Behavioral responses of the patient and family: myocardial infarction and coronary artery bypass surgery. In: Underhill SL, Woods SL, Froelicher ES, Halpeny CJ, editors. Cardiac nursing. 2. ed. Philadelphia: J.B. Lippincont; 1989. p.692-703. 
11. Jaarsma $T$, Kastermans M, Dassen $T$, Philipsen $H$. Problems of cardiac patients in early recovery. J Adv Nurs 1995; 21(1):21-7.

12. Newton KM, Froelicher ESS. Life-style adjustments. In: Underhill SL, Woods, SL, Froelicher, ES, Halpeny, CJ, editors. Cardiac nursing. 2. ed. Philadelphia: J.B. Lippincont; 1989. p.715-38.

13. DeBusk RF, Miller NH, Superko HR, Dennis CA, Thomas RJ, Lew $\mathrm{HT}$, et al. A case-management system for coronary risk factor modification after acute myocardial infarction. Int Crit Care Nurs 1994; 1120(9):721-9.

14. Legato MJ, Padus, E, Slaugter, E. Women's perception of their general health, with special reference to their risk of coronary artery disease: results of a national telephone survey. J Women's Health 1997; 6(2):189-98.

15. van Elderen-van Kemenade T, Maes S, van den Broek Y. Effects of a health education programme with telephone follow-up during cardiac rehabilitation. Br J Clin Psychol 1994; 33:367-78.

16. Nicklin WM. Post discharge concerns of cardiac patients as presented via a telephone callback system. Heart \& Lung 1986; 15(3):268-72.

17. Corsetti AM, Perry D. A comprehensive approach to facilitating the recovery of cardiac surgery patients. J Cardiovasc Nurs 1998; 12(2):82-90.

18. Hartz LK, Frantz RA. Characteristics of postoperative patienteducation programs for open-heart surgery patients in the United States. Heart \& Lung 1977;6(2):137-42.

19. Moore S.S. Effects of interventions to promote recovery coronary artery bypass surgical patients. J Cardiovasc Nurs 1997; 12(1):5970.

20. King KB, Parrinello KA. Patient perceptions of recovery from coronary artery bypass grafting after discharge from the hospital. Heart \& Lung 1988; 17(6):708-15.

21. Lovvorn J. Coronary artery bypass graft surgery: helping patients cope with postop problems. Am J Nurs 1982; 7:1073-5.

22. Dantas RAS. Reabilitação de pacientes após cirurgia de revascularização do miocárdio. [tese]. Ribeirão Preto (SP): Escola de Enfermagem de Ribeirão Preto/USP; 1999.

23. Ministério da Saúde (BR). Resolução n.1, de 13 de junho de 1988. Normas de pesquisa em saúde. Brasília (DF): Ministério da Saúde; 1998. Cap.2, p. 4-7: Aspectos éticos da pesquisa em seres humanos.
24. Thomas TA, Taylor SM, Crane MM, Cornett WR, Langan EM, Snyder BA. An analysis of limb-threatening lower extremity wound complications after 1090 consecutive coronary artery bypass procedures. Vasc Med 1999; 4(2): 83-8.

25. Wimmer Greinecker G, Matheis G, Brieden M, Dietrich M, Oremek G, Westphal K, et al. Neuropsychological changes after cardiopulmonary bypass for coronary artery bypass grafting. Thorac Cardiovasc Surg 1998; 46(4):207-12.

26. Bogdan R, Biklen S. Investigação qualitativa em educação: uma introdução à teoria e aos métodos. Portugal: Porto Editora; 1994.

27. Grady KL, Buckley DJ, Cisar NS, Fink NM, Ryan SD. Patient perception of cardiovascular surgical patient education. Heart \& Lung 1988; 17(4):349-55.

28. Meyer R, Latz, P. What open heart surgery patients want to know. Am J Nurs 1979; 79:1558-60.

29. Tack BB, Gilliss CL. Nurse-monitored cardiac recovery: a description of the first 8 weeks. Heart \& Lung 1990; 19(5):492-9.

30. Brunner LS, Suddarth DS. Tratado de enfermagem médicocirúrgica. 5.ed. Rio de Janeiro (RJ): Interamericana; 1985. p.543-62. 31. Begges VL, Willis SB, Maislen EL, Stokes TM, White D, Sanford $\mathrm{M}$, et al. Patient education for discharge after coronary artery bypass surgery in 1990s: are patients adequately prepared? J Cardiovas Nurs 1998; 12(4):72-86.

32. Jickling JL, Graydon JE. The information needs at time of hospital discharge of male and female patients who have undergone coronary artery bypass grafting: a pilot study. Heart \& Lung 1997; 26(5):350-7. 33. Oliveira MFP de, Sharousky LL, Ismael SMC. Aspectos emocionais no paciente coronariano. In: Oliveira MFP de, Ismael SMC, organizadoras. Rumos da psicologia hospitalar. São Paulo (SP): Papirus; 1995 . p. 185-97.

34. Lukkarinen, H. Quality of life in coronary artery disease. Nurs Res 1998; 47(6):337-43.

35. Newton KM, Killien MG. Patient/spouse learning needs after coronary artery bypass. Circulation 1986; 74(Suppl 2):291.

36. Marshall J, Penckofer S, Llewellyn J. Structured postoperative teaching and knowledge and compliance of patients who had coronary artery bypass surgery. Heart \& Lung 1986; 15(1):76-82 . 